\title{
Simulation of Structural Phenomena in Mixed-Particle Fluidized Beds
}

\author{
Kevin D. Seibert and Mark A. Burns \\ Dept. of Chemical Engineering, University of Michigan, Ann Arbor, MI 48109
}

\begin{abstract}
A statistical mechanical simulation was developed and used to predict the macroscopic and microscopic behavior of fluidized beds. In particular, the model was used to study structural phenomena in liquid fluidized beds that contain a distribution of particle sizes and/or densities. Bed structural phenomena, such as density-based and sizebased segregation, classification, expansion, inversion, and solids dispersion have all been successfully simulated. Experimental data for the segregation of glass beads of different sizes and different densities agree well with predictions made from the simulation (without adjustable parameters). Classification and dispersion simulations also show good agreement with experimental data.
\end{abstract}

\section{Introduction}

Most studies on the macroscopic properties of liquid fluidized beds have focused on predicting the expansion characteristic or the superficial fluid velocity-voidage relationship using the system's physical properties and operating conditions. While these models are accurate for fluidized beds of uniformly sized particles, the presence of different particle sizes and/or densities may affect both the expansion behavior and structure of the bed.

Various models have been proposed to describe structural phenomena such as segregation, mixing, and layer compositions in binary particle systems (Di Felice, 1993; Gibilaro et al., 1985; Juma and Richardson, 1979, 1983; Dutta et al., 1988; Kennedy and Bretton, 1966; Asif and Petersen, 1993). Experimental and empirical results for the pressure and concentration profiles that exist in binary systems have been used to make predictions of the solids concentration in segregated systems. Kennedy and Bretton (1966) and Al-Dibouni and Garside (1979) predicted binary segregation using a model that matched the diffusive and convective fluxes of each component. Other phenomena such as layer inversion have also been studied (Moritomi et al., 1982; Van Duijn and Rietema, 1982; Epstein and LeClair, 1985; Matsuura and Akehata, 1985; Gibilaro et al., 1986; Syamlal and O'Brien, 1988; Di Felice et al., 1988; Jean and Fan, 1986; Patwardhan and Tien, 1985).

Correspondence concerning this article should be addressed to M. A. Burns

Current address of K. D. Seibert: Merck and Co., Inc., P.O. Box 2000, RY50D-207, Rahway, NJ 07065 .
An example of a structural phenomenon seen in a mixedparticle liquid-fluidized bed is classification. Classification is the result of the stable fluidization of particles with nonuniform particle diameters and/or densities. The changing hydrodynamic forces on the particles cause them to segregate or "classify" with larger particles gravitating toward the bottom of the bed and smaller particles toward the top. Classified beds are currently used industrially in the isolation of adsorbing solutes (Gailliot et al., 1990; Draeger and Chase, 1990; Chase and Draeger, 1992a,b; Chase, 1994; Batt et al., 1995). Knowledge of the fluidization characteristics, the operating parameters, and the physical properties of the particles/bed is important in this type of application in order to maintain the stable classified structure.

Mixing, dispersion, and stable fluidization are phenomena that occur in distributed particle systems as well as in uniformly sized particle systems (Juma and Richardson, 1983; Di Felice, 1993; Avidan and Yerushalmi, 1985; van der Meer et al., 1984; Asif and Petersen, 1993). Studies of stable fluidization (De Luca et al., 1994) have included particle systems where the density of the particles is very close to that of the fluidizing media, and situations where high fluid velocities (large void fractions) are used.

In order to understand the various structural phenomena present in fluidized beds, we have modeled mixed-particle beds using a discrete particle simulation technique previously developed for uniformly sized particle systems (Seibert and Burns, 1996). The simulation we have developed is ideal for 
systems that include distributions of particle sizes and/or densities. The method is analogous to a Monte Carlo (MC) simulation for molecular systems; the forces affecting the motion of individual particles are calculated and used to predict macroscopic bed properties. The advantage of an MC-type simulation lies in its ability to account for specific particle-size distributions in such calculations. An additional advantage of these simulations is the rich visual information that can be obtained including exact particle locations and spatially resolved structural phenomena.

The primary difficulty in including distributed particle sizes and densities in such force calculations is the difficulty in calculating the hydrodynamic force on a particle. In fluidized beds containing uniformly sized particles, the variation in hydrodynamic force as a function of position in the bed can be calculated using the local void fraction. For distributed particle systems, the surrounding particles' sizes and densities also affect these calculations

\section{Model Description}

\section{Simulation operation}

The model development is based on the simulation previously developed for a uniform particle system (Seibert and Burns, 1996). Simulations are performed with a small representative number of particles in a simulation volume (Figure 1). The simulation volume consists of a three-dimensional space with fully periodic boundary conditions in the $\mathrm{X}$ and $\mathrm{Z}$ directions, and a solid bottom and infinite height in the $Y$ direction. The width of the simulation space was 6-20 particle diameters, and the depth was a minimum of 10 particle diameters.

Typically, a simulation is initiated by randomly placing $\sim 10^{3}$ particles into the simulation space, and generating random particle coordinates in a nonoverlapping configuration. Prior to any changes in an operational parameter, simulations are run for $\sim 10^{4}$ moves/particle until a steady-state configuration is reached. The move distance of a particle is

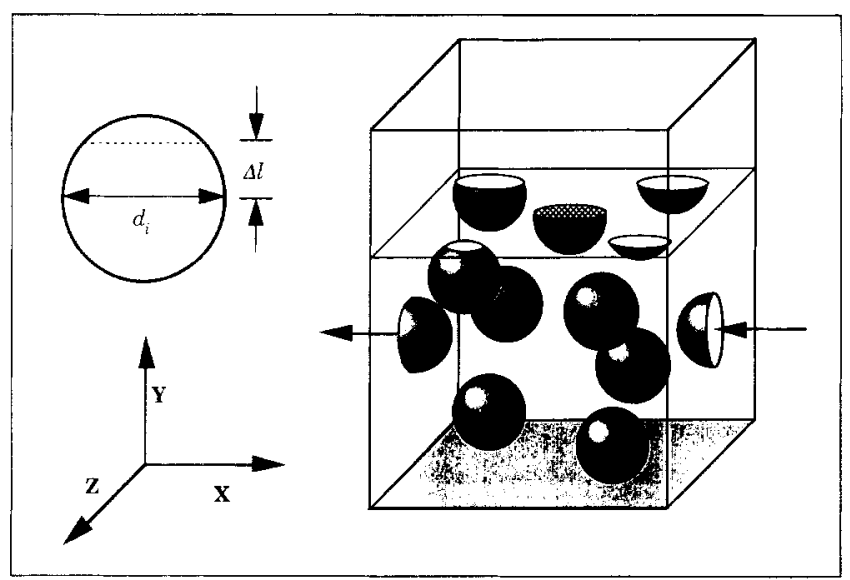

Figure 1. Example of simulation space.

The simulation space has periodic boundaries in the $X$ and $Z$ direction, a solid bottom, and infinite height in the $Y$ direction. Local void fraction is calculated by passing a plane through the center of the selected particle (crosshatched) and computing the contribution to the intersected plane area from each intersected particle. The tops of the particles have been removed to show the intersected area. adjusted to maximize the number of accepted moves. Typically, a move distance equal to $10 \%$ of a particle diameter results in the most rapid approach to equilibrium (Seibert and Burns, 1996). Run times are on the order of $10^{7}$ to $10^{8}$ attempted particle moves after any change to operational parameters (fluid or particle physical properties, fluid velocity, and so on) is made.

Particle movement begins with the random selection of a single particle in the representative system. The selected particle is moved a fixed distance $S$ in a random direction in three dimensions. If the movement of the particle results in an overlap with an adjacent particle, the selected move is automatically rejected and the particle is returned to the original coordinates. Nonoverlapping and energetically favorable moves (moves that would result in a lowering of the system energy) are accepted with $100 \%$ probability.

For an energetically unfavorable nonoverlapping move, a random probability $\left(p_{r}\right)$ between 0 and 1 is calculated along with the change in the system's energy $\Delta E$ resulting from the move. A move is accepted if $p_{r}$ is less than $p_{a}$, where $p_{a}$ is given by

$$
p_{a}=e^{-(\Delta E) / K E}
$$

and $K E$ is the kinetic energy of the particle. A declined move resets the selected particle's coordinates. Once such a single particle move has been completed, the process (selection, movement, overlap check, and acceptance) is repeated until the system converges to a steady-state configuration. Steady state is determined by an absence in change in the overall system void fraction and in the macroscopic bed structure.

The two quantities in Eq. $1, \Delta E$ and $K E$, must be calculated independently. To compute the kinetic energy $(K E)$ of the particle, it has been shown (Buyevich, 1994; Nettleton, 1993) that the kinetic energy may be represented by the product of the particle mass and the velocity of the particle relative to the fluid

$$
K E=\frac{1}{2} m_{p} U^{2}
$$

where $m_{p}$ is the mass of the particle and $U$ is a characteristic particle velocity. For this study, we have used the interstitial fluid velocity of the fluid through the particle media as the characteristic velocity. Other forms of $K E$ that can be used are discussed elsewhere (Seibert and Burns, 1996).

\section{Calculation of net force}

To compute the change in system energy $(\Delta E)$, the net force on a particle is multiplied by the vertical distance the particle has traveled. The difficulty in this calculation is determining the exact force on an individual particle. Di Felice (1995) provides a review of the hydrodynamics of fluidization and an excellent description of the interaction forces in a fluid-particle system to aid in this determination.

In a fluidized bed at steady state, the hydrodynamic force $\left(F_{f}\right)$ is balanced by the gravity and buoyancy forces $\left(F_{g, b}\right)$

$$
F_{f}=F_{g, b}=\left(\rho_{p}-\rho_{f}\right) V_{p} g
$$


where $V_{p}$ is the volume of a particle, $g$ is the acceleration due to gravity, and $\rho_{f}$ and $\rho_{p}$ are the densities of the fluid and particle, respectively. The hydrodynamic force $F_{f}$ is a sum of two flow-derived forces acting on the particle: a pressure force caused by the flow-induced pressure gradient (porous media) and a viscous drag force caused by fluid shear at the particle's surface

$$
F_{f}=F_{d}+F_{P}=F_{d}+V_{p}(\partial P / \partial z)
$$

These two forces can be grouped together as

$$
F_{f}=V_{h}(\partial P / \partial z)
$$

where $\partial P / \partial z$ is the pressure gradient in the bed and $V_{h}$ is the fictitious volume that when multiplied by the pressure gradient would give the appropriate hydrodynamic force.

The pressure gradient in a fluidized bed at steady state is equivalent to the weight of the bed in the fluid divided by the cross-sectional area. For a fluidized bed containing only one type of particle, the pressure drop is given by

$$
\frac{\partial P}{\partial z}=(1-\epsilon)\left(\rho_{p}-\rho_{f}\right) g
$$

By substituting this relationship and the relationship describing $F_{f}$ (Eq. 3) into Eq. 5, $V_{h}$ can be shown to be $V_{p} /(1-e)$. The hydrodynamic force as a function of the flow-induced pressure gradient is then

$$
F_{f}=\frac{\partial P}{\partial z} \frac{V_{p}}{(1-\epsilon)}
$$

Deriving an expression for the hydrodynamic force in a fluidized bed with a distribution of particle sizes and densities requires an analogous approach, but with a modification of the pressure gradient calculations. The pressure gradient in a mixed bed system resulting from the weight of the particles and cross-sectional area is then given by

$$
\frac{\partial P}{\partial z}=\left[\phi_{1}\left(\rho_{1}-\rho_{f}\right)+\phi_{2}\left(\rho_{2}-\rho_{f}\right)+\phi_{3}\left(\rho_{3}-\rho_{f}\right)+\cdots\right] V_{p} g
$$

and is a function of all particle densities $\left(\rho_{i}\right)$ and concentrations $\left(\phi_{i}\right)$. Again, combining this equation with Eqs. 3 and 5 yields a final form for the hydrodynamic force on a particle in a mixture of other particles based on the induced pressure gradient

$$
F_{f}=\frac{\partial P}{\partial z} \frac{\left(\rho_{p}-\rho_{f}\right)}{\sum_{i=1}^{n} \phi_{i}\left(\rho_{i}-\rho_{f}\right)} V_{p}
$$

Thus, if the pressure gradient is known along with the composition of the bed $\left(\phi_{i}\right)$, the force on any particle can be calculated.
Several predictions for the pressure gradient in porous media have been developed including empirical and semi-empirical models (Richardson and Zaki, 1954; Foscolo et al., 1983), which are based on the Hagen-Poiseuille tube and annulus flow (Joshi, 1983), and theoretical predictions based on unit cell models and solutions to the Navier-Stokes equations (Barnea and Mizrahi, 1973; Batchelor, 1972; Reed and Anderson, 1980; Happel, 1958; Kuwabara, 1959; Moritomi et al., 1985). For this work, we have focused on a pressure drop model that uses purely theoretical solutions to the equations of motion around particles in a fluidized bed.

Happel's solution to the equations of motion around a random assemblage of spheres (Happel, 1958) provides the necessary pressure gradient information. Happel's model for the force on a particle has been shown to yield good predictions for the expansion characteristic of particles in liquid fluidized beds operating at low particle Reynolds numbers. The advantage of using a unit cell model is that it allows us to make predictions of fluidized-bed behavior from a purely predictive approach, where no adjustable parameters are necessary.

The pressure gradient derived from Happel's model is given by the following equation:

$$
\frac{\partial P}{\partial z}=f(\epsilon) \frac{18 \mu(1-\epsilon)}{\langle d\rangle^{2}} U_{s}
$$

where $\mu$ is the viscosity of the solution, $\langle d\rangle$ is the average diameter of the particles, and $f(\epsilon)$ is a voidage function given by the following equation:

$$
f(\epsilon)=\frac{\left(3+2 \gamma^{3}\right)}{\left(3-(9 / 2) \gamma+(9 / 2) \gamma^{5}-3 \gamma^{6}\right)}
$$

where

$$
\gamma=(1-\epsilon)^{1 / 3}
$$

The original equation was modified to account for a mixed particle system by using the average particle diameter (Tien, 1989; Gibilaro et al., 1986). In our case, we define $\langle d\rangle$ as the equivalent hydraulic diameter of the mixture in the immediate area around the particle (Carmen, 1956; Gibilaro et al., 1986) or

$$
\frac{1}{\langle d\rangle}=\frac{1}{1-\epsilon}\left(\frac{\phi_{1}}{d_{1}}+\frac{\phi_{2}}{d_{2}}+\frac{\phi_{3}}{d_{3}}+\cdots\right)
$$

$\langle d\rangle$ is a function of the particle sizes, solids fractions, and local void fraction around the selected particle.

Combining Eqs. 3, 5, 8, and 10 yields an expression for the hydrodynamic force on a particle in a flow field

$$
F_{f}=\frac{\left(\rho_{p}-\rho_{f}\right)}{\sum_{i=1}^{n} \phi_{i}\left(\rho_{i}-\rho_{f}\right)} f(\epsilon)(1-\epsilon) 3 \pi \mu U_{s} \frac{d_{p}^{3}}{\langle d\rangle^{2}}
$$

where $U_{s}$ the superficial fluid velocity. Note that $f(\epsilon)$ and $\langle d\rangle$ are defined by Eqs. 11 and 13, respectively. Also, $\pi d^{3} / 6$ 
was substituted for $V_{p}$. The net force on a particle that is used in the simulation to predict particle motion is given by the difference between the equilibrium value of the hydrodynamic force (that is, $F_{g, b}$ ) and the calculated value at the current conditions or

$$
F_{\imath}=F_{f}-F_{g, b}
$$

These equations were used to simulate fluidized-particle systems from a purely predictive approach with no adjustable parameters. In this approach, we have included the fundamental forces that affect particle motion (buoyancy, pressure gradient, gravity, and viscous drag) but have neglected second-order effects. Forces that arise from excess mixing and nonuniform flow distribution in a fluidized bed or other body forces (electrostatic, magnetic) could be added to the simulation. Also, multibody hydrodynamic interactions (that is, the deviation of the hydrodynamic force from Eq. 14 due to flow disturbances) could be added.

\section{Experimental Studies}

\section{Materials and equipment}

Fluidization runs were performed using $25^{\circ} \mathrm{C}$ distilled/deionized water as the fluidizing medium. Data was taken in 1.5 cm ID Kontes Flex-Column chromatography columns. The columns were fitted with a porous distributor, $0.32 \mathrm{~cm}$ thick, with pore sizes ranging from $25-50 \mu \mathrm{m}$. The fluid was pumped using a model QD laboratory pump from Fluid Metering, Inc. for fluidization data. Data were obtained by fluidizing nickel spheres from Johnson Matthey, white and blue high index glass beads, and white low index beads from Potters Industries. The simulation program used for configuration generation, spatial resolution, and position analysis was written in FORTRAN and Absoft's MacFortran II for the Macintosh. Computer trials were run on SUN Sparc 20 computers. Particle distribution information was calculated from projected area measurements with Biological Detection Systems Image (Ver. 1.2) analysis software, a Macintosh IIfx computer, a Hammamatsu camera, and a Zeiss Axioscop microscope.

\section{Methods}

Soldis Fraction Data. Solids fraction data were taken by adding a known, dry mass of particles to a graduated chromatography column and allowing the particles to settle; initial bed depths ranged from 4 to $8 \mathrm{~cm}$. Initially, acetone was pumped through the column to reduce bubble formation on the surface of the glass particles. The feed solution was changed to distilled/deionized water and was pumped up through a porous distributor at the bottom of the column and through the bed of particles. After the particles had completely segregated and reached an equilibrium bed height, the flow was immediately reversed, and a plug of particles formed at the bottom of the bed. This plug was pushed out of the bed, and $0.5-\mathrm{cm}$ sections were sliced off of the plug. These samples were dried and the nickel removed using a magnet. The mass of the two samples were then weighed with an analytical balance. Solids fraction is computed using the packed bed void fraction, initial independent bed heights of the two layers, and expanded bed heights of the two layers, assuming the layers expand independently. The solids fraction as a function of position may then be determined from the relative amounts of solids present in the fraction.

Particle Data. The average particle diameter was determined using six hundred particles from each particle type. Particles were randomly chosen, and the average diameters were calculated using the Sauter mean particle diameter of the distribution. Particle density was calculated by weighing a volume of particles in a volumetric flask. Water at $25^{\circ} \mathrm{C}$ was added to the flask, and the flask was shaken to remove any air bubbles. The density of the particles is then calculated from the known mass of particles, the difference in the flask volume, and the known volume of water present (calculated from the mass of the water added to the flask and the density of the water at that temperature).

\section{Results and Discussion}

\section{Segregation}

The segregation of two particle types was studied with a mixture of $150 \mu \mathrm{m}$ (gray) and $200 \mu \mathrm{m}$ (white) glass beads, both with a density of $2.45 \mathrm{~g} / \mathrm{cm}^{3}$. Experimental results for the segregation of these two different particle types are shown in Figure 2. Initially, the smaller (gray glass) beads are placed at the bottom of the bed (Figure 2a) and the larger (white glass) beads are layered on top. As the flow of water is initiated $(0.60 \mathrm{~cm} / \mathrm{s})$, the bed expands and the layers begin to mix (Figure 2b). Finally, the layers reach their steady-state configuration with the larger particles concentrated at the bottom of the fluidized bed and the smaller, darker particles near the top of the bed (Figure 2c).

By simulating a fluidized bed using the same set of conditions, we find similar results for the final predicted steadystate bed structure. Figures $3 \mathrm{a}$ and $3 \mathrm{~b}$ show the initial and final configurations for the simulation of the fluidization of the same 150 and $200 \mu \mathrm{m}$ glass beads at the same superficial fluid velocity. As can be seen by the figure, the particles, which are initially completely mixed (they are initially placed in this random configuration), separate into two distinct layers of particles based solely on their different sizes.

Results for the inclusion of a difference in density between two types of particles are similar to those obtained from size based segregation experiments. Simulation and experimental results for the fluidization of a mixture of $150-\mu \mathrm{m}$ glass beads, half (by volume) of which have a density of $2.45 \mathrm{~g} / \mathrm{cm}^{3}$ and the other half with a density of $4.5 \mathrm{~g} / \mathrm{cm}^{3}$, are shown in Figure 4 . At steady state, both the simulation and the experiments proceed to a configuration with two distinct particle layers with the more dense particles (white) located toward the bottom of the bed and the less dense particles (black) toward the top of the bed (Figures $4 \mathrm{~b}$ and $4 \mathrm{c}$ ). Note that if the particles are initially placed in the fluidized bed with the less dense below the more dense and the fluid is introduced to the column, the layers change places with the more dense particles moving to the bottom of the bed.

\section{Classification}

Segregation of two particle types into layers is simply a special case of classification of particle sizes that occurs in a system that contains a full distribution of particle sizes. Ex- 


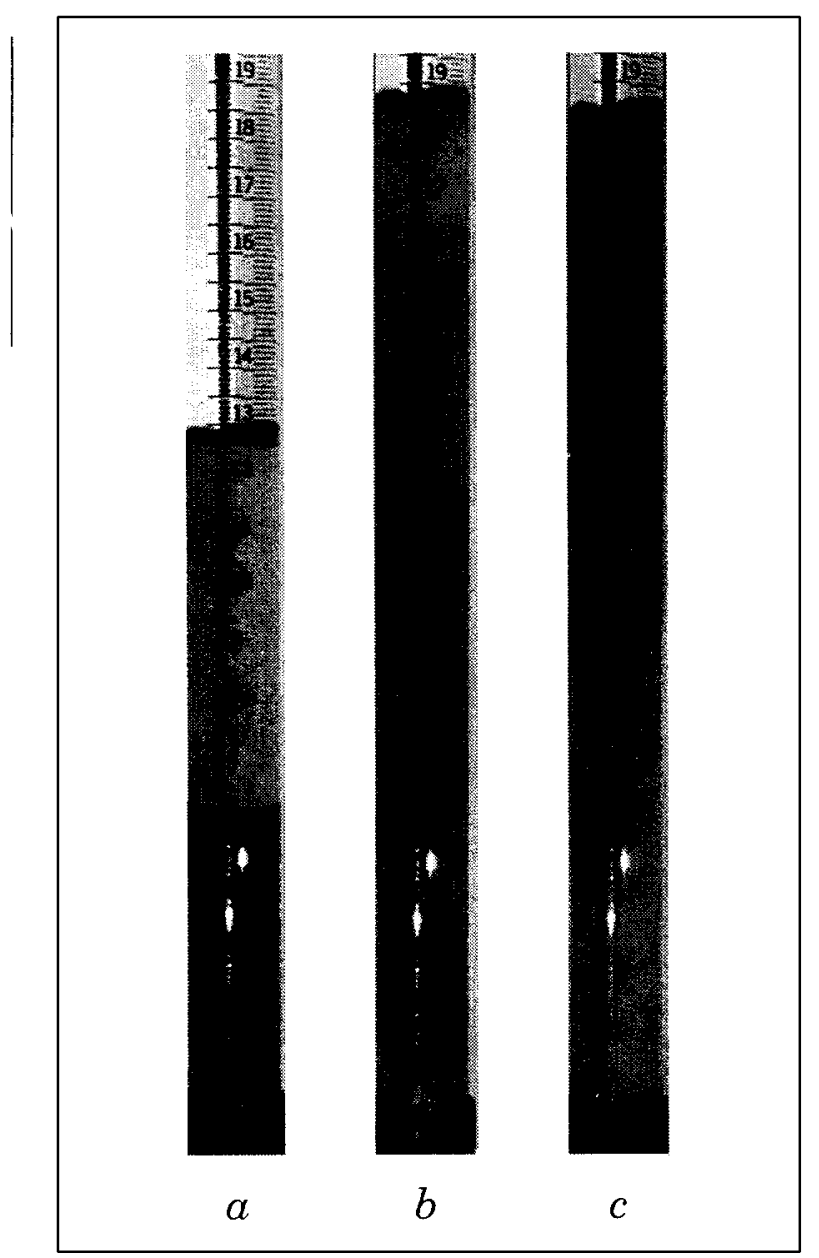

Figure 2. Experimental results for segregation of particles layers in water.

(a) Particles are placed in a bed with the smaller particles (150- $\mu \mathrm{m}$ glass, $\rho=2.45 \mathrm{~g} / \mathrm{cm}^{3}$ ) below the larger particles (200- $\mu \mathrm{m}$ glass, $\left.\rho=2.45 \mathrm{~g} / \mathrm{cm}^{3}\right)$; (b) the fluid is then introduced into the column, and the bed completely mixes; (c) the bed eventually segregates with the larger (white) particles concentrated in the bottom of the bed and the smaller (gray) particles concentrated in the top of the bed.

perimentally, we find if a distribution of particles sizes are present in a fluidized bed the smaller particles tend to accumulate toward the top of the bed and the larger particles accumulate toward the bottom. The reduction in solids mixing due to the presence of a particle-size distribution, also known as hydrodynamic stabilization, is used industrially in several fluidized-bed applications (Gailliot et al., 1990; Draeger and Chase, 1990; Batt et al., 1995).

We have run simulations that include a distribution of particle densities and sizes to visualize particle classification. Figure 5a shows the steady-state configuration for a system containing $400 \mu \mathrm{m}$ particles with a distribution of densities ranging from 2.0 and $2.9 \mathrm{~g} / \mathrm{cm}^{3}$. Particles with the mean density $\left(2.4 \mathrm{~g} / \mathrm{cm}^{3}\right)$ have been labeled to allow the visualization of the classification and the focusing of a certain density particle in a single location in the bed. Based upon the particle's relative density, this group of particles tends to focus in the center region of the bed: above the denser particles but below the less dense ones.
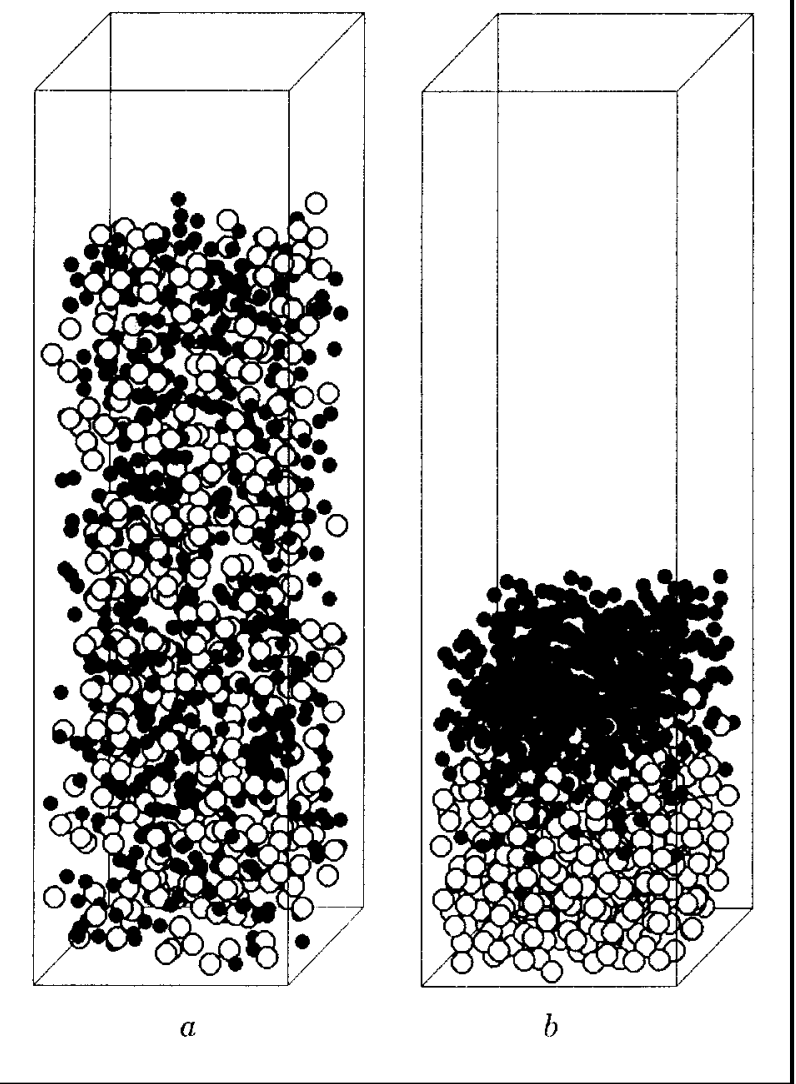

Figure 3. Initial and final configurations for size-based segregation.

Initially, 1,000 particles are placed into the simulation space, half of which are $200-\mu \mathrm{m}$ glass spheres, density $=4.5 \mathrm{~g} / \mathrm{cm}^{3}$, and half are $150-\mu \mathrm{m}$ spheres with the same density. By specifying a superficial fluid velocity $\left(U=0.6 \mathrm{~cm} / \mathrm{s}, \quad \rho_{f}=1.0\right.$ $\mathrm{g} / \mathrm{cm}^{3}, \mu=0.01 \mathrm{cp}$ ) where both species are fluidized, we are able to visualize the segregation of these two species into distinct layers of particles. The smaller species accumulates toward the top of the fluidized bed and the larger concentrates toward the bottom.

Figure 5b shows this same classification phenomenon, but based upon particle size rather than density. Fluidization of particles with a density of $2.45 \mathrm{~g} / \mathrm{cm}^{3}$ and a size distribution ranging from $310-490 \mu \mathrm{m}$ are simulated at a velocity of 0.8 $\mathrm{cm} / \mathrm{s}$. As in the case of density-based segregation, and similar to what we find experimentally, the particles tend to focus in layers based upon their relative sizes. Additionally, we find that when the particles with the system mean diameter are labeled, they are also focused toward the center of the bed.

\section{Expansion characteristic}

Simulating the expansion characteristic of uniformly sized particle systems has been previously investigated (Seibert and Burns, 1996). By operating the simulation using the mean particle diameter of the system, it is possible to make predictions of the expansion characteristic of a specific fluid-particle system. Including the full particle-size distribution in the simulation, we have the capability to predict the expansion characteristic of complicated distributions of particles. 


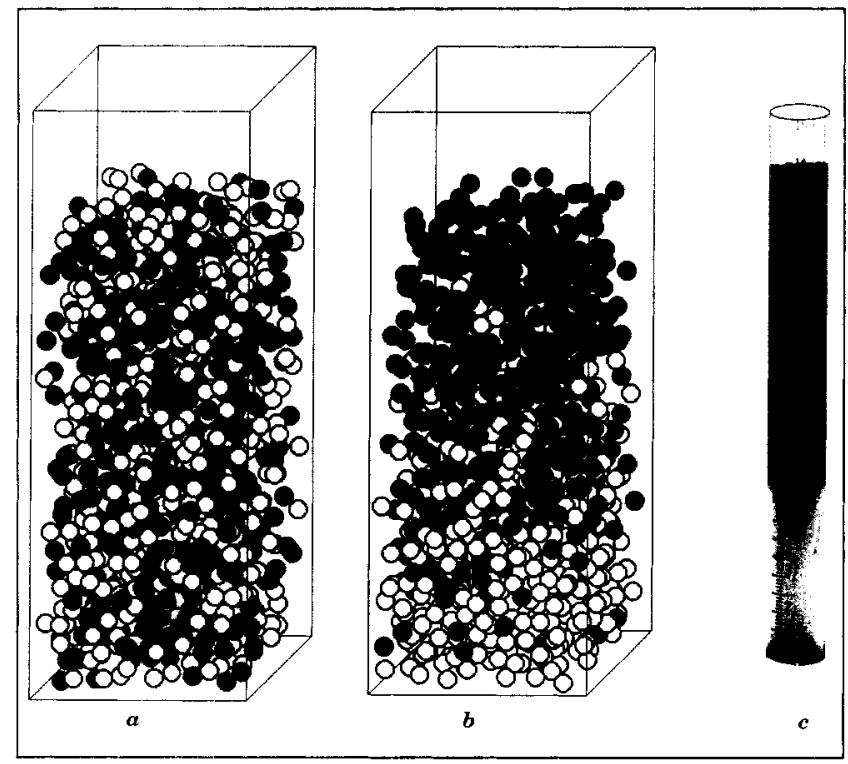

Figure 4. Initial and final simulation configurations, and experimental results for density-based segregation.

(a) Initially 1,000 particles $(150 \mu \mathrm{m})$ are placed into the simulation space, half (by volume) of which have a density of $4.5 \mathrm{~g} / \mathrm{cm}^{3}$, and half with a density of $2.45 \mathrm{~g} / \mathrm{cm}^{3}$; (b) at a superficial fluid velocity of $0.40 \mathrm{~cm} / \mathrm{s}$, both species are fluidized, and segregation of the two particle types occurs. The less dense particles (labeled black) expand to a larger void fraction and accumulate toward the top of the fluidized bed, while the more dense particles concentrate at the bottom; (c) experimental verification of this segregation using white glass particles $\left(150 \mu \mathrm{m}, \rho=4.5 \mathrm{~g} / \mathrm{cm}^{3}\right)$ and gray glass particles $\left(150 \mu \mathrm{m}, \rho=2.45 \mathrm{~g} / \mathrm{cm}^{3}\right)$ shows excellent agreement between experimental data and simulated bed structure.

Figure 6 shows the particle-size distribution for narrowly distributed and bimodally distributed particle systems. The two distributions have identical mean particle sizes $(286 \mu \mathrm{m}$ particles, $\left.\rho=2.45 \mathrm{~g} / \mathrm{cm}^{3}\right)$; therefore, an empirical model based upon mean particle sizes would predict the same expansion characteristic for both distributions. Figure 7 shows the simulation predictions and experimental expansion curves for the distributions in Figure 6. The simulation predicts the bimodal distribution to expand to a greater degree than the relatively narrow distribution with the same mean particle diameter. As can be seen by the figure, inclusion of only the basic hydrodynamic forces allows the simulation to predict the experimentally observed difference between narrowly distributed and bimodal particle-size distributions.

\section{Particle dispersion}

The liquid fluidization of two particle types results in the segregation of particles into layers in most cases. At the interface between the two particle types, the particle layers tend to mix slightly and form a mixed region of particles between the two monocomponent zones. This mixing is driven by a variety of factors including the stability of the supporting particle layer, the inconsistencies in the flow field resulting in nonuniform fluidization, and the two layers' physical properties. In our simulation we are able to investigate how the random motion of particles and their physical properties may

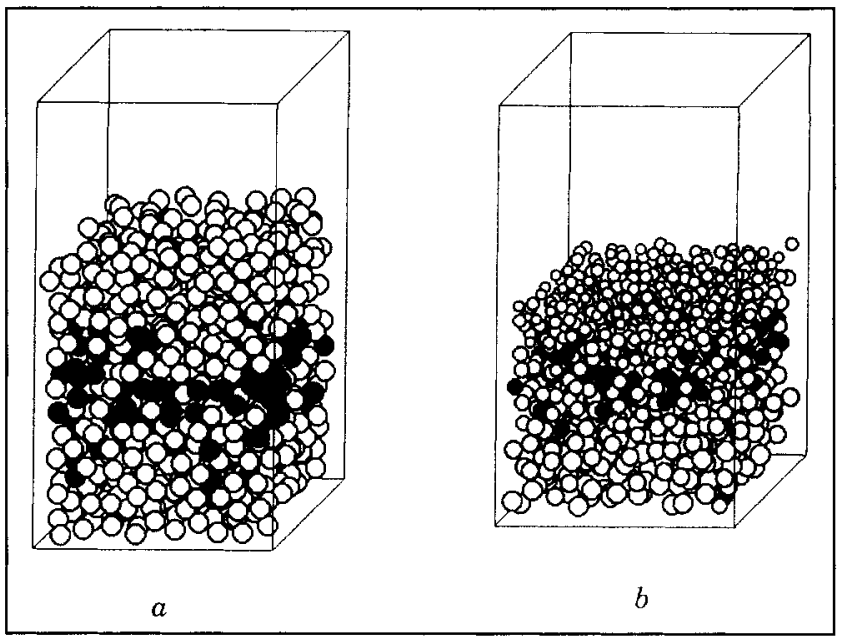

Figure 5. Classification based upon density and size.

Simulations were performed with 1,000 particles and a distribution of particle densities and sizes. (a) A system of 400 $\mu \mathrm{m}$ particles with densities ranging from 2.0 to $2.9 \mathrm{~g} / \mathrm{cm}^{3}$ are allowed to come to a steady-state configuration at a superficial fluid velocity of $0.6 \mathrm{~cm} / \mathrm{s}$. The particles having a density equal to $2.4 \mathrm{~g} / \mathrm{cm}^{3}$ (the system average) are filled in black. As can be seen by the figure, the particles classify into layers according to the density of the particles present in the system; (b) particles with a density of $2.45 \mathrm{~g} / \mathrm{cm}^{3}$ and a size distribution ranging from $310 \mu \mathrm{m}-490 \mu \mathrm{m}$ were simulated at a superficial fluid velocity of $0.8 \mathrm{~cm} / \mathrm{s}$. In this case, classification is again observed but the classification is due to size rather than density differences.

lead to the penetration of particle layers in a segregated fluidized bed.

We have experimentally measured the concentration profile of two solids as a function of axial position in a binary-solid fluidized bed and have compared these solids con-

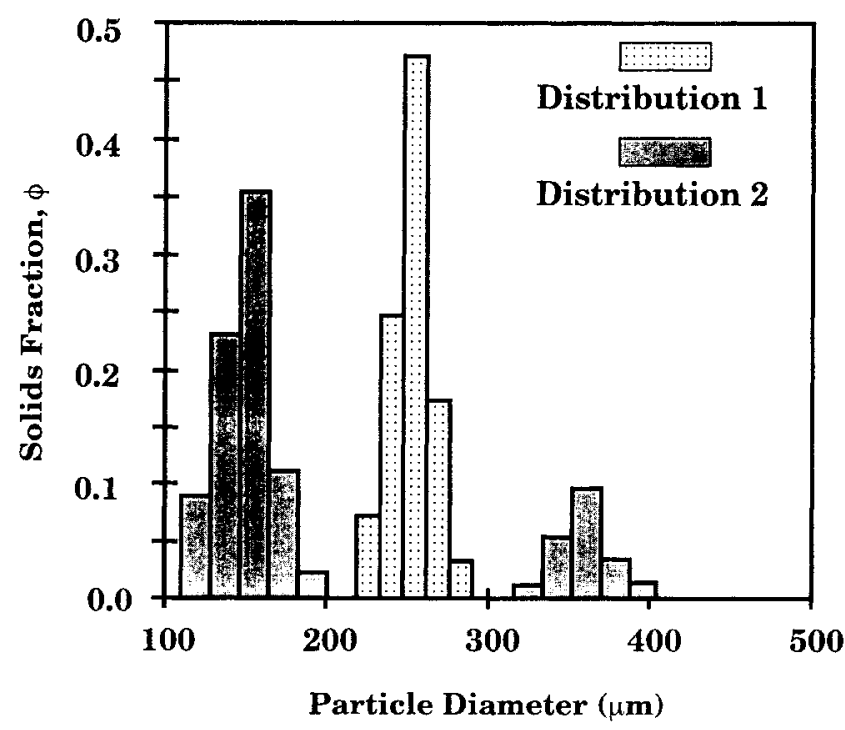

Figure 6. Particle-size distribution with the same volume average particle diameter.

A distribution of particles is developed that has a bimodal distribution (No. 2), but still has the same average particle diameter as a normal distribution (No. 1). Traditional expansion models would predict the same expansion characteristic for both systems. 


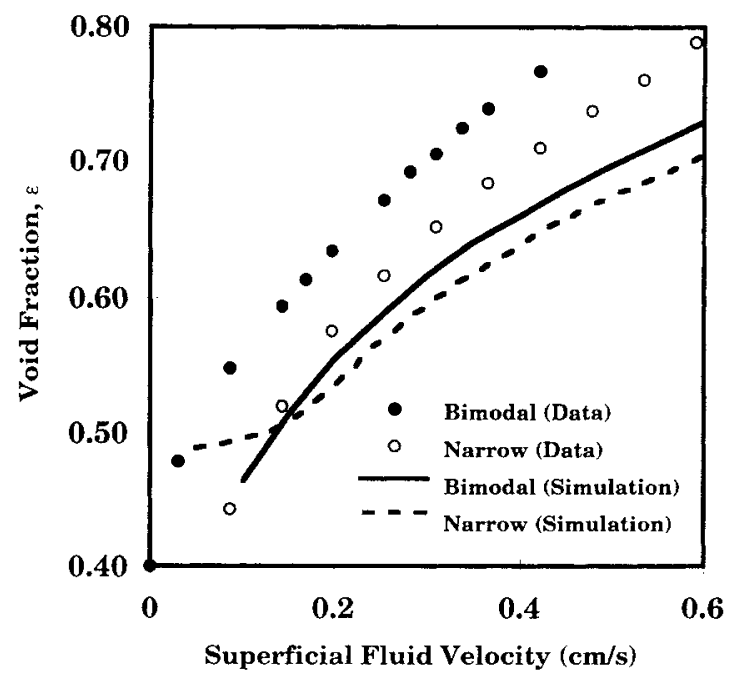

Figure 7. Simulation of the expansion characteristic of two particles systems with different distributions and the same mean particle size.

This figure shows the expansion characteristic predicted by the simulation for the distributions shown in Figure 6. The simulation predicts a significant increase in the void fraction as a function of velocity for the bimodal distribution. Experimental data for the same system shows the same trend with the experiments showing a larger void fraction at identical velocities for the bimodal distribution over the narrowly distributed system.

centration profiles to those that we predicted from the simulation. $75 \mu \mathrm{m}$ nickel $\left(\rho=8.65 \mathrm{~g} / \mathrm{cm}^{3}\right)$ was fluidized with a $115 \mu \mathrm{m}$ glass $\left(\rho=2.45 \mathrm{~g} / \mathrm{cm}^{3}\right)$ and allowed to completely segregate and reach a steady-state configuration. The bed was then collapsed and analyzed for solids concentration as a function of location in the bed. These results were repeated for two superficial fluid velocities, $0.218 \mathrm{~cm} / \mathrm{s}$ and $0.327 \mathrm{~cm} / \mathrm{s}$. Figure 8 shows the experimental data and the simulation results for the prediction of the solids fraction as a function of the dimensionless position $(\mathrm{y} / \mathrm{L})$ in the fluidized beds. As can be seen by the figure, the simulation predicts a sharp interface between the two particle phases. Experimentally, we find nearly identical results with slightly more mixing between the two layers found experimentally for this nickel-glass system.

In addition to the nickel-glass system, we have compared simulation results to experimental data points from Juma and Richardson (1983) for a binary particle system of 1.9 and 2.98 $\mathrm{mm}$ glass beads $(\rho=2.96)$ fluidized in paraffin oil. Figure 9 shows the experimental results and the simulated concentration profiles. These results show good agreement between the simulated concentration profiles and experimental data for this paraffin oil-glass bead system.

The profiles in Figures 8 and 9 reveal two difficulties encountered when simulating mixed particle systems. First, when the sizes of the two different particle types become significantly different, the length of the simulation runs increases dramatically. Figure 8 shows concentration profiles for larger glass beads with smaller nickel particles. In order to simulate this mixed system, the simulation space is set at only six glass particles in width. While this size is small for the glass particles, the bed is quite large for the smaller nickel particles and therefore requires a large number of nickel par-
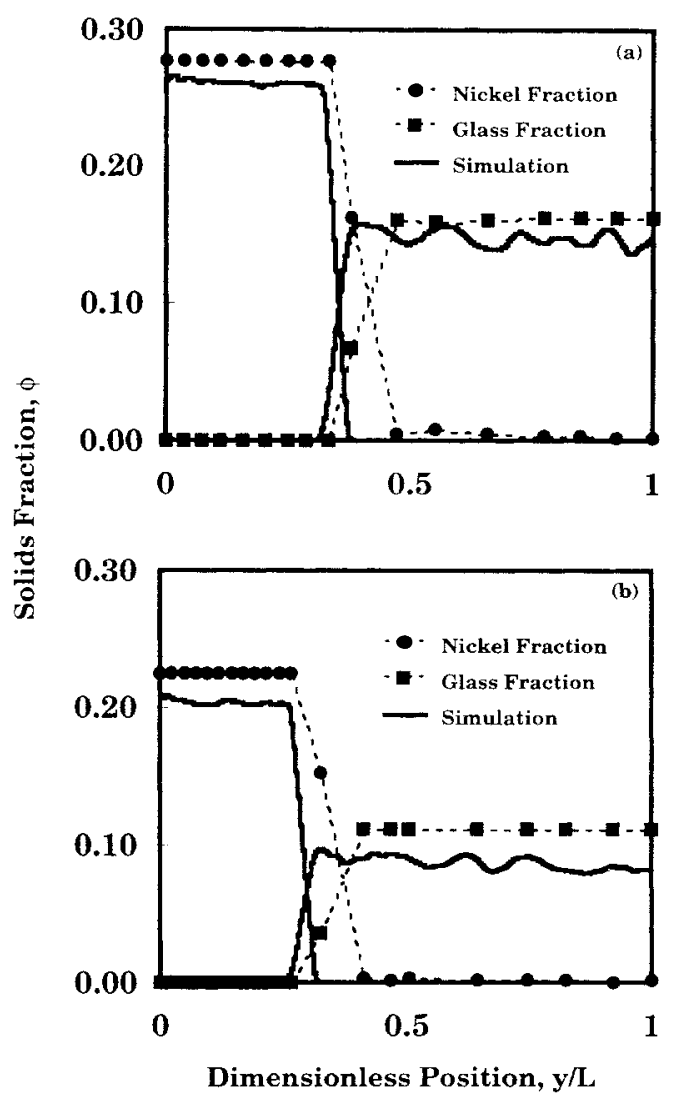

Figure 8. Simulation of the concentration profiles in a segregated binary particle system.

Good agreement is found between the experimental data and the simulated concentration profiles for both velocities we simulated. (a) $U_{s}=0.218 \mathrm{~cm} / \mathrm{s}$; (b) $U_{s}=0.327 \mathrm{~cm} / \mathrm{s}$.

ticles to form a bed of any significant depth. The very long run times that result make simulating such systems very difficult.

Second, the small simulation size relative to the larger particle results in periodicity effects. The periodic oscillations seen in the glass concentration profile in Figure 8 occurs whenever the width and/or height of the simulated bed is less than 5 or 10 particle diameters. The nickel bed, which contains many more particles, shows none of these oscillations. Note that the concentration profile of the larger glass beads in Figure 9 also has some oscillations.

In addition to the mixing in segregated particle layers predicted by the simulation, we have looked at the differences found in simulating classification and layer formation in liquid and gas fluidized systems. In general, gas fluidized beds have been shown experimentally not to classify by particle size. By simulating a fluidized bed of glass particles using both a liquid $\left(\rho=1.0 \mathrm{~g} / \mathrm{cm}^{3}, \mu=1.0 \mathrm{cP}, U_{s}=0.632 \mathrm{~cm} / \mathrm{s}\right)$ and a gas $\left(\rho=0.0182 \mathrm{~g} / \mathrm{cm}^{3}, \mu=0.0013 \mathrm{cP}, U_{s}=0.586 \mathrm{~m} / \mathrm{s}\right)$, we found similar results (Figure 10). Although the model equations predict both beds to expand to an identical void fraction $(0.65)$, we find that the mean particle size (labeled black in Figure 10) is more concentrated in the center of the bed in the liquid fluidized bed than the gas fluidized bed. Note that these simulations were performed from two different sets of initial conditions (a randomly mixed expanded system and a 


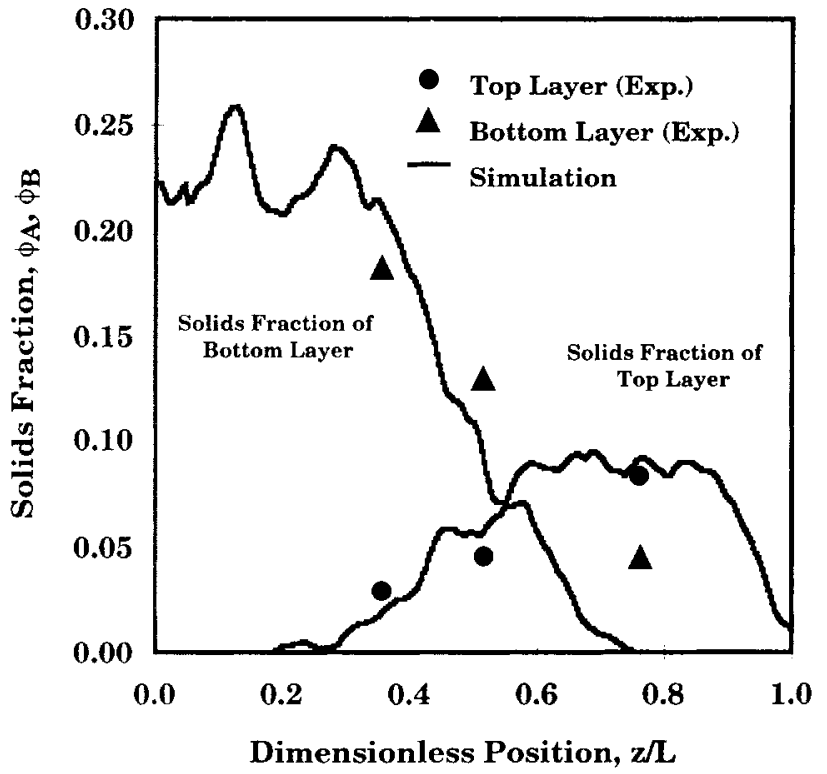

Figure 9. Simulation of mixing and dispersion of layers in segregated, binary particle systems.

The simulated concentration profiles shown are for $0.19 \mathrm{~cm}$ and $0.298 \mathrm{~cm}$ glass beads $\left(\rho=2.96 \mathrm{~g} / \mathrm{cm}^{3}\right)$. These are compared to the experimental data of Juma and Richardson (1983). Good quantitative and qualitative agreement between experimental data and simulation predictions is found although the simulation does slightly underpredict the layer mixing.

completely segregated packed system) to insure that the results were not simply due to incomplete simulation times.

\section{Particle layer inversion}

When combining particle types of different sizes and densities in a fluidized bed, complicated structural phenomena may result. Particulate systems that contain a mixture of higher density smaller particles and lower density larger particles may exhibit the phenomenon of particle layer inversion (Richards and Locke, 1940). At low velocities, the smaller particle species will concentrate toward the bottom of a fluidized bed and the larger less dense particles toward the top. As the superficial fluid velocity is increased, both layers of particles expand until reaching the inversion velocity. At the inversion velocity, both particle types coexist as a single mixed layer. Further increasing the fluid velocity results in the layer of smaller particles passing through the layer of large particles and remaining above them. A great deal of experimental and modeling studies have been undertaken to explain this phenomenon of binary layer inversion (De Felice, 1995).

We have simulated this phenomenon of inversion using our discrete particle simulation. By simulating the same experimental system investigated by Moritomi et al. (1985) and Gibilaro et al. (1986) (Figure 11), we find an identical inversion behavior, where, at lower velocities the smaller particles ( $163 \mu \mathrm{m}$ glass beads, $\rho=2.45 \mathrm{~g} / \mathrm{cm}^{3}$ ) tend to accumulate toward the bottom of the bed while at higher velocities, the small particles pass through the larger particle layer $(775 \mu \mathrm{m}$ coal char, $\rho=1.38 \mathrm{~g} / \mathrm{cm}^{3}$ ) and orient themselves above the larger particle layer.

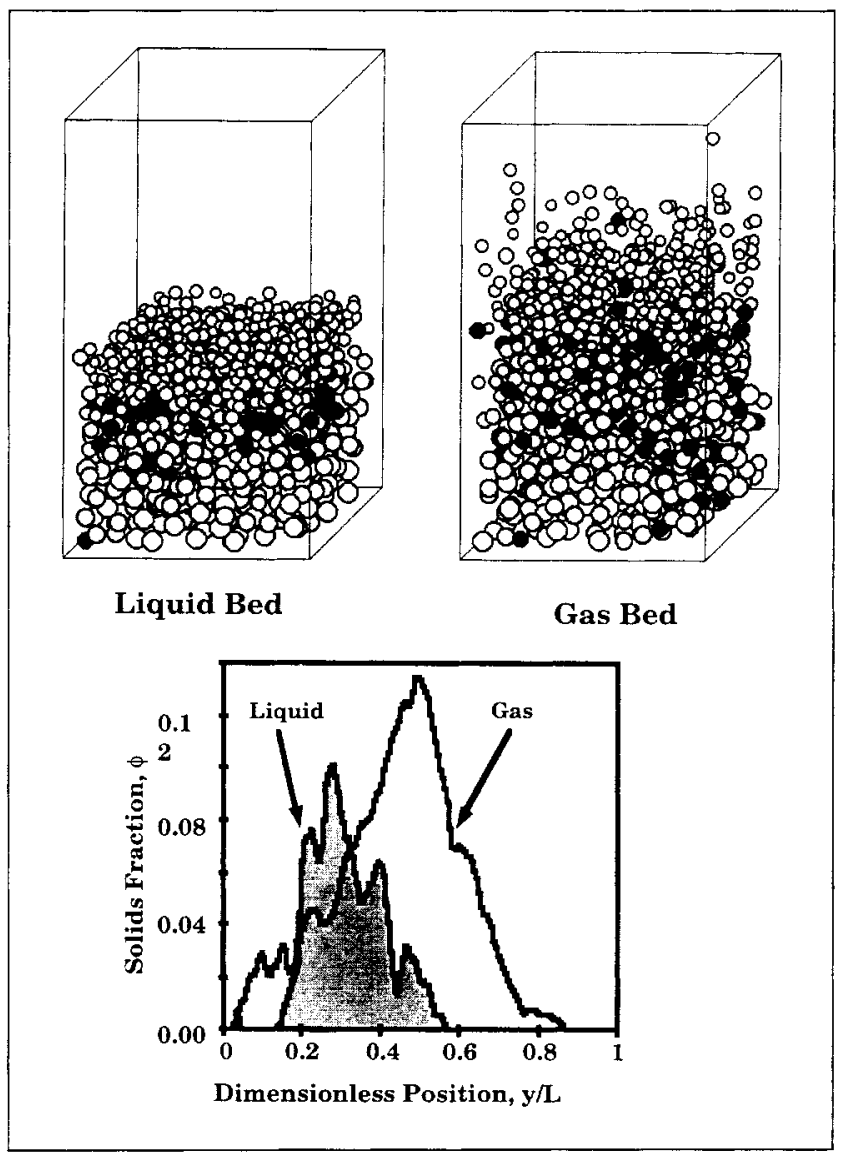

Figure 10. Simulation of mixing in liquid- and gas-fluidized beds.

The simulation of liquid-fluidized beds shows a significantly greater degree of classification and focusing of particle types in a particular region of the bed. The solids fraction profile of the mean particle size shows that the gas-fluidized bed has particles of a single type distributed almost throughout the entire simulated bed.

\section{Conclusions}

In summary, we have developed a discrete particle simulation that applies the concept of statistical mechanics and random motion to particulate behavior. We have taken this simulation, previously developed for uniformly sized particles, and extended the operation to distributed particle systems. We have shown this simulation to be suitable for the simulation of macroscopic expansion characteristics, while still allowing for the visualization of the various microscopic structural phenomena that occur in a fluidized system. Although we did see differences in the quantitative predictions for expansion prediction, the accuracy of the prediction is limited to the accuracy of the hydrodynamic model (Seibert and Burns, 1996).

Using this simulation, we have been able to predict structural phenomena such as segregation, classification, inversion, and penetration of particle layers in multiparticle fluidization. When simulating fluidized-bed structure, it is important that detailed consideration be given to the proper determination of the forces on a particle and the energy associated with particle motion. In addition to the forces we have included in this simulation, electrostatic, magnetic, or fric- 

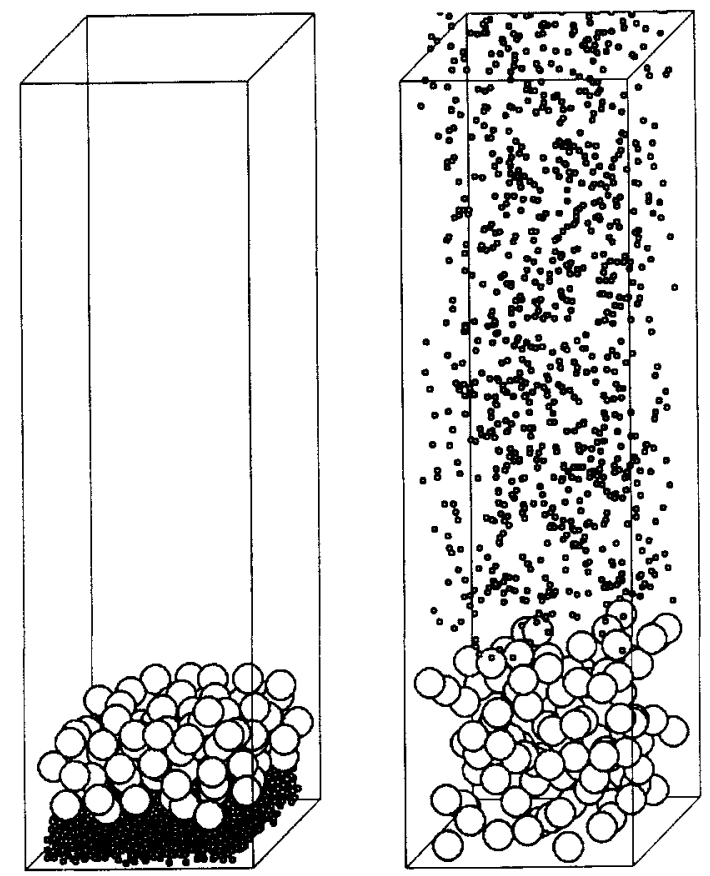

Figure 11. Simulation of inverting particle layers in a binary solid system.

At low velocities, the smaller particles $(163-\mu \mathrm{m}$ glass, $\rho=$ $2.45 \mathrm{~g} / \mathrm{cm}^{3}$ ) in the system tend to focus toward the bottom of the fluidized bed. As the velocity is gradually increased through the inversion velocity, the particles change locations and the smaller particles reorient themselves above the larger particles $\left(775-\mu \mathrm{m}\right.$ coal, $\left.\rho=1.38 \mathrm{~g} / \mathrm{cm}^{3}\right)$

tional interparticle forces can be added to the simulation to calculate the effect of these forces on the macroscopic, as well as microscopic structural behavior of a fluidized bed.

\section{Acknowledgments}

The financial support of the University of Michigan, Dept. of Chemical Engineering, the National Science Foundation and the Donors of the Petroleum Research Fund (administered by the American Chemical Society) is gratefully acknowledged.

\section{Notation}

$L=$ bed length

$p_{a}=$ acceptance probability

$\stackrel{P}{\Delta P}=$ pressure drop

\section{Subscripts}

$$
\begin{aligned}
d & =\text { drag } \\
P & =\text { pressure } \\
t & =\text { total }
\end{aligned}
$$

\section{Literature Cited}

Al-Dibouni, M. R., and J. Garside, "Particle Mixing and Classification in Liquid Fluidized Beds," Trans. Instn. Chem. Engrs., 57, 94 (1979).

Asif, M., and J. N. Petersen, "Particle Dispersion in a Binary SolidLiquid Fluidized Bed," AIChE J., 39, 1465 (1993).

Avidan, A., and J. Yerushalmi, "Solids Mixing in an Expanded Top Fluid Bed," AIChE J., 31, 835 (1985).
Barnea, E., and J. Mizrahi, "A Generalized Approach to the Fluid Dynamics of Particulate Systems, Part 1. General Correlation for Fluidization and Sedimentation in Solid Multiparticle Systems," Chem. Eng. J., 5, 171 (1973).

Batchelor, G. K., "Sedimentation in a Dilute Polydispersed System of Interacting Spheres," J. Fluid Mech., 52, 245 (1972).

Batt, B. C., V. M. Yabannavar, and V. Singh, "Expanded Bed Ad sorption Process for Protein Recovery from Whole Mammalian Cell Culture Broth," Bioseparation, 5, 41 (1995).

Buyevich, Y. A., "Fluid Dynamics of Coarse Dispersions," Chem. Eng. Sci., 49, 1217 (1994).

Carmen, P. C., Flow of Gases through Porous Media, Butterworth Scientific Publications, London (1956).

Chase, H. A., and N. M. Draeger, "Affinity Purification of Proteins Using Expanded Beds," J. of Chromatography, 59, 129 (1992a).

Chase, H. A., and N. M. Draeger, Sep. Sci. and Technology, 27, 2021 (1992b).

Chase, H. A., "Purification of Proteins by Adsorption Chromatography in Expanded Beds," Tibtech, 296 (1994).

De Luca, L., D. Hellenbroich, N. J. Titchener-Hooker, and H. A. Chase, "A Study of the Expansion Characteristics and Transient Behavior of Expanded Beds of Adsorbent Particles Suitable for Bioseparations," Bioseparation, 4, 311 (1994).

Di Felice, R., "Mixing in Segregated, Binary-Solid Liquid-Fluidised Beds," Chem. Eng. Sci., 48, 881 (1993).

Di Felice, R., "Hydrodynamics of Liquid Fluidisation," Chem. Eng. Sci., 50, 1213 (1995).

Di Felice, R., L. G. Gibilaro, and P. U. Foscolo, "On the Inversion of Binary-Solid Liquid Fluidised Beds," Chem. Eng. Sci., 43, 979 (1988).

Draeger, N. M., and H. A. Chase, I. Chem. Eng. J. Symp. Ser., 18, 161 (1990)

Dutta, B. K., S. Bhattacharya, S. K. Chaudhury, and B. Barman, "Mixing and Segregation in a Liquid Fluidized Bed of Particles with Different Size and Density," Can. J. Chem. Eng., 66, 676 (1988)

Epstein, N., and B. P. LeClair, "Liquid Fluidisation of Binary Particle Mixtures. II. Bed Inversion," Chem. Eng. Sci, 40, 1517 (1985).

Foscolo, P. U., L. G. Gibilaro, and S. P. Waldram, "A Unified Model for Particulate Expansion of Fluidised Beds and Flow in Fixed Porous Media," Chem. Eng. Sci., 38, 1251 (1983).

Gailliot, F. P., C. Gleason, J. J. Wilson, and J. Zwarick, "Fluidized Bed Adsorption for Whole Broth Extraction," Biotechnol. Prog., 6, 370 (1990)

Gibilaro, L. G., I. Hossain, and S. P. Waldram, "On the Kennedy and Bretton Model for Mixing and Segregation in Liquid Fluidized Beds," Chem. Eng. Sci., 40, 2333 (1985).

Gibilaro, L. G., R. Di Felice, S. P. Waldram, and P. U. Foscolo, "A Predictive Model for the Equilibrium Composition and Inversion of Binary-Solid Liquid Fluidized Beds," Chem. Eng. Sci., 41, 379 (1986).

Happel, J., "Viscous Flow in Multiparticle Systems: Slow Motion of Fluids Relative to Beds of Spherical Particles," AIChE J., 4, 197 (1958).

Jean, R., and L. S. Fan, "On the Criteria of Solids Layer Inversion in a Liquid-Solid Fluidized Bed Containing a Binary Mixture of Particles," Chem. Eng. Sci., 41, 2811 (1986).

Joshi, J. B., "Solid-Liquid Fluidised Beds: Some Design Aspects," Chem. Eng. Res. Des., 61, 143 (1983).

Juma, A. K. A., and J. F. Richardson, "Segregation and Mixing in Liquid Fluidized Beds," Chem. Eng. Sci., 38, 955 (1983).

Juma, A. K. A., and J. F. Richardson, "Particle Segregation in Liquid-Solid Fluidised Beds," Chem. Eng. Sci., 34, 137 (1979).

Kennedy, S. C., and R. H. Bretton "Axial Dispersion of Spheres Fluidized with Liquids," AIChE J., 12, 24 (1966).

Kuwabara, S., "The Forces Experienced by Randomly Distributed Parallel Circular Cylinders or Spheres in a Viscous Flow at Small Reynolds Numbers," J. Phys. Soc. Jap., 14, 527 (1959).

Matsuura, A., and T. Akehata, "Distribution of Solid Particles and Bed Expansion in a Liquid Fluidized Bed Containing a Binary Mixture of Particles," Paper C-108, Mtg. of Soc. of Chem. Engrs. of Japan, Yokohama (1985).

Moritomi, H., T. Yamagishi, and T. Chiba, "Prediction of Complete Mixing of Liquid-Fluidized Binary Solid Particles," Chem. Eng. Sci., 41, 297 (1985) 
Moritomi, H.. T. Iwase, and T. Chiba, "A Comprehensive Interpretation of Solid Layer Inversion in Liquid Fluidised Beds," Chem. Eng. Sci., 37, 1751 (1982).

Nettleton, R. E., "On the Relation between Thermodynamic Temperature and Kinetic Energy per Particle," Can. J. Phys., 72, 106 (1993).

Patwardhan, V. S., and Chi Tien, "Sedimentation and Liquid Fluidization of Solid Particles of Different Sizes and Densities," Chem. Eng. Sci., 40, 1051 (1985).

Reed, C. C., and J. L. Anderson, "Hindered Settling of a Suspension at Low Reynolds Number, AIChE J., 26, 816 (1980).

Richards, R. H., and C. E. Locke, Textbook of Ore Dressing, McGraw-Hill, London (1940).

Richardson, J. F., and W. N. Zaki, "Sedimentation and Fluidisation: I," Trans. Inst. Chem. Eng., 32, 35 (1954).
Seibert, K. D., and M. A. Burns, "Simulation of Fluidized Beds and Other Fluid Particle Systems Using Statistical Mechanics," AIChE J., 42, 660 (1996).

Syamlal, M., and T. J. O'Brien, "Simulation of Granular Layer Inversion in Liquid Fluidized Beds," Int. J. of Multiphase Flow, 14, 473 (1988)

Tien, Chi, Granular Filtration of Aerosols and Hydrosols, Butterworths Publishers, Stoneham, MA (1989).

van der Meer, A. P., C. M. R. J. P. Blanchard, and J. A. Wesselingh, "Mixing of Particles in Liquid Fluidised Beds," Chem. Eng. Res. Des., 62, 214 (1984).

Van Duijn, G., and I. Rietema, "Segregation of Liquid-Fluidised Solids," Chem. Eng. Sci., 37, 727 (1982).

Manuscript received Jan. 9, 1997, and revision received Nov. 18, 1997. 